\title{
Simulation-based Procedural Skills Maintenance Training for Neonatal- Perinatal Medicine Faculty
}

\author{
Taylor Sawyer $^{1}$, Thomas Strandjord ${ }^{2}$ \\ 1. Department of Pediatrics, University of Washington 2. Pediatrics, University of Washington School of \\ Medicine
}

$\square$ Corresponding author: Taylor Sawyer, tlsawyer@uw.edu

Disclosures can be found in Additional Information at the end of the article

\section{Abstract}

Introduction: Some procedures in neonatal-perinatal medicine are rarely performed in day-today clinical practice. Relying on clinical exposure alone to maintain procedural competency in those skills may be inadequate. Simulation-based training provides an option to practice these rarely performed procedures in order to maintain competency. Here, we report the preliminary results of a simulation-based procedural skills maintenance curriculum for neonatal-perinatal medicine faculty.

Methods: Based on a needs assessment survey, we identified several procedures that faculty neonatologists believed would benefit from simulation-based maintenance training. During a two hour simulation session, faculty were divided into small groups and rotated through four separate procedural skills stations: pericardiocentesis, electrocardioversion, defibrillation, and exchange transfusion. Using a collaborative learning model, each group worked as a team to practice the procedural skills. Pre- and post-session surveys were used to evaluate the educational impact of the sessions on both self-perceived knowledge and procedural competency.

Results: Participants reported significant improvements in self-perceived procedural competency in three of the four procedural skills. The majority of participants reported feeling more confident in their ability to perform the procedures after participation in simulation. The overall quality of the event was rated as 'good' or 'very good' by all participants. All participants 'agreed' or 'strongly agreed' that they learned something new during the simulation session.

Discussion: Simulation-based maintenance training for rarely performed procedures in neonatal-perinatal medicine is feasible, and was associated with improvements in self-

Received 04/21/2014 Review began 04/22/2014 Review ended 04/23/2014 Published 04/23/2014

\section{C) Copyright 2014}

Sawyer et al. This is an open access article distributed under the terms of the Creative Commons Attribution License CC-BY 3.0., which permits unrestricted use, distribution, and reproduction in any medium, provided the original author and source are credited. perceived competency amongst neonatal faculty members. Based on these early results, further investigations are planned.

Categories: Medical Education, Medical Simulation, Pediatrics

Keywords: procedural skills, simulation, skill maintenance, faculty, neonatal, perinatal

\section{Introduction}

Competency in procedural skills is a critical component of neonatal-perinatal medicine. The traditional model of maintaining competency in procedural skills is through the ongoing performance of procedures during day-to-day clinical care. However, a subset of essential 
procedures are rarely performed in the neonatal intensive care unit. Additionally, in an academic setting, faculty physicians are often not afforded the opportunity to perform procedures due presence of residents, fellows, pediatric hospitalists, and advanced practice nurses. This makes relying on day-to-day clinical practice to provide essential experience for skill maintenance particularly difficult for academic neonatal-perinatal faculty.

Simulation-based training in procedural skills is a well-accepted method of procedural skill training [1-4]. Simulation can also be used for procedural skills maintenance [5-6]. Therefore, a simulation-based procedural skills maintenance curriculum may provide a viable option for neonatal-perinatal medicine faculty members to maintain procedural competency in rarely performed procedures.

Here, we report the preliminary results of a simulation-based procedural skills maintenance curriculum for rarely performed procedures in neonatal-perinatal medicine. The aim of this pilot project was to determine the feasibility of conducting a simulation-based skills maintenance training session in an academic neonatal division, and to obtain preliminary data on learning outcomes. Our hypothesis was that participation in the simulation session would be associated with improvements in self-perceived knowledge and competency with rarely performed procedural skills amongst our academic faculty.

\section{Materials And Methods}

In order to determine which procedural skills should be included in a skill maintenance curriculum, we performed a needs assessment survey. The survey was created using SurveyMonkey.com and was emailed to all faculty in the Division of Neonatal-Perinatal Medicine at the University of Washington School of Medicine. Based on the results of the needs assessment survey, four procedures were identified for inclusion in the first session: pericardiocentesis, electrocardioversion, defibrillation, and exchange transfusion.

The simulation session was conducted in place of a regularly scheduled division academic conference. During the simulation session, neonatal faculty was divided into four small groups of three to four members. Each group rotated through four procedural skills stations, with each station dedicated to a single procedure. At each station, an applicable mannequin, or task trainer, was available for the group to work with. Medical equipment and materials required for the procedure were also available at each station. Informational handouts were provided at each station that could be referenced by the groups, if needed. As this was a skills maintenance session, not a training session, there was no external facilitation or coaching of the faculty. Instead, the faculty group members were responsible for working as a team to properly set up the equipment and ensuring that each team member completed the procedural skill. No grading or scoring of procedural skill was conducted in this initial session. Each of the four groups spent 30 minutes at each of the four procedural skills stations. The entire session lasted two hours.

Pre- and post-session surveys were used to collect demographic data and to evaluate the educational impact of the session. Pre-session questionnaires were emailed to participants one week in advance of the simulation session. The pre-session questionnaire included demographic data (age, gender, etc.) and asked participants to report their prior experience with each of the procedures (total number completed in career and last time completed). Postsession questionnaires were emailed to participants the day after the workshop. Reminders were sent until all participants completed the post-session questionnaire. The questionnaires asked participants to rate their own personal knowledge and competency in each of the procedural skills. Self-perceived knowledge of the indications and contraindications of performing each procedure was rated on a 1 to 5 Likert-type scale, with $1=$ 'very poor', $2=$ 'poor', 3 = 'fair', 4 = 'good', and 5 = 'very good'. Self-perceived level of competency in performing each procedure was rated on a 1 to 5 Likert-type scale, with $1=$ 'not competent', $2=$ 'somewhat 


\section{Cureus}

competent', 3 = 'uncertain', 4 = 'competent', and 5 = 'highly competent'. Post-session surveys also asked participants to rate if they learned something new by participating in this simulation-session on a five point Likert-type scale ( 1 = 'strongly disagree', to 5 = 'strongly agree'), and if they would like to participate in another simulation session in the future. Participants were also asked to rate the overall quality of the simulation event on a scale from 1 to 5 ( 1 = 'very poor', to 5 = 'very good').

Demographic data were analyzed using descriptive statistics. Changes in self-perceived knowledge and competency on the pre- and post-session surveys were analyzed using Student's t-test, or Mann-Whitney Rank Sum Test, as applicable. A P value of $<0.05$ was considered statistically significant. Statistical analysis was performed using SigmaPlot 11 (Systat Software Inc.). This study was deemed exempt from Institutional Review Board review.

\section{Results}

Eleven faculty participated in the first simulation session. Pre- and post-session surveys were received from all 11 faculty members (100\% response rate). Demographic data on the participants is provided in Table 1 . The average age of the participants was 48 years old, with a range from 36 to 63 . The majority of participants (63\%) were within 10 years of graduation from fellowship. However, $36 \%$ of participants were > 20 years from completion of training.

Age, years

$30-40$

$41-50$

$51-60$

$61-70$

Years since completion of fellowship

$1-5$

6-10

$11-15$

$16-20$

$>20$

Gender

Male

Female

\section{TABLE 1: Demographic Data}




\section{Cureus}

Survey results on the procedural experience of the participants are provided in Table 2. A majority of participants had never performed pericardiocentesis or defibrillation on a neonate in clinical practice (55\% and $72 \%$, respectively). Over a quarter (27\%) had never performed electrocardioversion. All faculty participants had previously performed an exchange transfusion. Of the faculty who had previously performed pericardiocentesis, electrocardioversion, and defibrillation, the majority had not performed the procedure in over two years, whereas the majority (54\%) of those same participants had performed an exchange transfusion within the past two years.

\section{Pericardiocentesis}

Times performed, N (\%)
$6(55)$

2 (18)

$1(9)$

$1(9)$

$1(9)$

6-10

$>10$

Time since last performed

Never performed
$<1$ month
$>1$ month, $<6$ months
$>6$ months, < 1 year
$>1$ year, < 2 years
$>2$ years
$>3$ years
$>5$ years

(55)

$3(27)$

$1(9)$

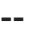

$3(27)$

1 (9)

$3(27)$
8 (72)

2 (18)

1 (9)

1 (9)

$3(27)$

1 (9)

1 (9)

4 (9)

8 (72)

$-$

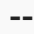

1 (9)

$-$

2 (18)

$3(27)$

$4(36)$

$-$

--

3 (27)

1 (9)

A summary of the self-perceived scores for knowledge and competency on the pre- and postsession surveys are provided in Table 3. The only improvement in self-perceived knowledge scores was in pericardiocentesis $(\mathrm{P}=0.04)$. There were significant improvements in selfperceived procedural competency in three of the four procedural skills (pericardiocentesis; $\mathrm{P}=$ 0.002, electrocardioversion; $\mathrm{P}=0.02$, and defibrillation; $\mathrm{P}=0.006$ ). 


\section{Cureus}

\begin{tabular}{|c|c|c|c|c|c|c|}
\hline & \multicolumn{3}{|c|}{ Knowledge $(n=11)$} & \multicolumn{3}{|c|}{ Competency $(n=11)$} \\
\hline & Pre-session & Post-session & $\mathbf{P}$ & Pre-session & Post-session & $\mathrm{P}$ \\
\hline Pericardiocentesis, mean (SD) & $3.5(0.9)$ & $4.3(0.5)$ & 0.04 & $2.3(1.3)$ & $3.8(0.8)$ & 0.002 \\
\hline Cardioversion & $3.6(0.9)$ & $4.3(0.5)$ & 0.05 & $3.1(1.3)$ & $4.2(0.4)$ & 0.02 \\
\hline Defibrillation & $3.5(0.8)$ & $4.1(0.5)$ & 0.08 & $2.7(1.1)$ & $3.9(0.3)$ & 0.006 \\
\hline Exchange transfusion & $4.6(0.5)$ & $4.7(0.5)$ & 0.68 & $4.5(0.5)$ & $4.4(0.5)$ & 0.70 \\
\hline
\end{tabular}

\section{TABLE 3: Self-Reported Scores for Knowledge and Competency}

Knowledge scores: 1 = very poor, $2=$ poor, $3=$ fair, 4 = good, 5 = very good

Competency scores: $1=$ not competent, $2=$ somewhat competent, $3=$ uncertain, $4=$ competent, $5=$ highly competent

Post-session surveys were universally positive regarding the educational experience. The overall quality of the event was rated as 'good' or 'very good' by all participants. All participants 'agreed' or 'strongly agreed' that they learned something new during the workshop. All participants 'agreed' or 'strongly agreed' that they would like to participate in similar events in the future.

\section{Discussion}

Preliminary data on a simulation-based procedural skills maintenance training session for academic neonatal-perinatal faculty members showed the training to be both feasible and educationally beneficial. The feedback received showed improvements in self-perceived competency in three of the four procedures included in the simulation session. The simulation training session was highly rated by the neonatal faculty members. All the faculty members expressed interest in participating in a similar event in the future. While multiple reports exist on the use of simulation-based education for pediatric residents and neonatal-perinatal fellows, to our knowledge, this report is the first to describe educational outcomes for a simulation-based procedural skills maintenance session for neonatal-perinatal faculty members.

Maintaining competency in procedural skills is an important part of medical practice, and is a vital component of the American Board of Medical Specialties core competencies of Patient Care and Procedural Skills and Practice-based Learning and Improvement [8]. Traditional methods of maintaining competency in procedural skills, such as exposure through ongoing clinical practice, may be inadequate for some rarely performed procedural skills because the incidence of performing some procedures during day-to-day clinical care is extremely limited. Our results showed that many of the faculty involved in the simulation session had never clinically performed some of the procedures included in the simulation session. For those who had previously performed the procedure clinically, there were significant time gaps reported since the last time the procedure was performed (greater than five years in some cases). These results provide a compelling case for the need to provide procedural skills maintenance training, which does not rely solely on clinical care experience. Simulation-based practice may provide the only viable option for this ongoing maintenance training. 
We believe the use of a needs assessment helped focus the simulation session on the procedures that were maximally beneficial for the faculty participants. Faculty in the simulation session reported the largest gains on self-perceived competency in those procedures in which they had the least clinical experience. The only procedure that was not associated with improvements in self-perceived competency, exchange transfusion, was the most commonly performed of the four procedures included in the simulation session. The needs assessment survey did not ask participants to provide their clinical experience with the procedures, but rather rate how much they would like to participate in simulation-based procedural skills practice on the procedures. It appears that the faculty members we surveyed naturally gravitated towards rarely performed procedures for inclusion, and rated more commonly performed procedures, such as endotracheal intubation and umbilical vessel catheterization, as much less desired. We theorize this was due to the significant prior clinical experience with these commonly performed skills amongst the faculty participants, and the perceived lack of educational benefit from practicing those procedures using simulation. This finding highlights the importance of targeting procedural skills training based on the specific needs of the learners. As the educational needs of faculty may significantly differ from those of residents or fellows, performing specific needs assessments of each learner group is important.

This pilot study has several limitations. The number of participants in the simulation session was low. With only 11 faculty participants, the generalizability of the results shown here is unclear. Larger studies using a multi-center approach would provide stronger data on the effects of the training. Another limitation is that participation in this simulation was not mandatory for faculty, and as a result, there may have been some selection bias on the part of the participants (e.g. only those who wanted to participate and enjoy simulation as a learning modality participated). Finally, the learning outcome measures reported are low on the Kirkpatrick Scale of Learning Outcomes, with a description of only the 'Reactions' (what participants thought and felt about the training) as opposed to Learning, Behavior, or Results [7]. Examining improvements in actual clinical care resulting from the simulation session would strengthen the results of this study. However, given the rarity of the procedures involved in the session, showing improvements during clinical care may be extremely difficult.

\section{Conclusions}

In conclusion, we performed a simulation-based procedural skills maintenance session for neonatal-perinatal faculty members. Faculty in our program reported improvements in selfperceived competency in three of four rarely performed procedures after completion of the simulation session. Simulation-based procedural skills maintenance training sessions may provide an acceptable method for faculty members to maintain procedural skills in rarely performed clinical procedures. Based on our preliminary results, we plan to conduct future simulation-based sessions on a semi-annual basis within our program. Our division is currently exploring making participation in procedural skills maintenance session mandatory for faculty as a way to ensure ongoing practice with rarely performed procedural skills.

\section{Additional Information}

\section{Disclosures}

Human subjects: All authors have confirmed that this study did not involve human participants or tissue. Animal subjects: All authors have confirmed that this study did not involve animal subjects or tissue. Conflicts of interest: In compliance with the ICMJE uniform disclosure form, all authors declare the following: Payment/services info: All authors have declared that no financial support was received from any organization for the submitted work. Financial relationships: All authors have declared that they have no financial relationships at present or within the previous three years with any organizations that might have an interest in the submitted work. Other relationships: All authors have declared that there are no other 


\section{Cureus}

relationships or activities that could appear to have influenced the submitted work.

\section{References}

1. Lammers R, Davenport M, Korley F, et al.: Teaching and assessing procedural skills using simulation: metrics and methodology. Acad Emerg Med. 2008, 15:1079-1087.

2. Kneebone R: Evaluating clinical simulations for learning procedural skills: a theory-based approach. Acad Med. 2005, 80:549-553.

3. McLeod PJ, Steinert Y, Trudel J, Gottesman R: Seven principles for teaching procedural and technical skills. Acad Med. 2001, 76:1080.

4. Wang E, Quinones J, Fitch M, et al.: Developing technical expertise in emergency medicinethe role of simulation in procedural skill acquisition. Acad Emerg Med. 2008, 15:1046-1057.

5. Kovacs G, Bullock G, Ackroyd-Stolarz S, Cain E, Petrie D: A randomized controlled trial on the effect of educational interventions in promoting airway management skill maintenance. Ann Emerg Med. 2000, 36:301-9.

6. Niles D, Sutton RM, Donoghue A, et al.: "Rolling Refreshers": A novel approach to maintain CPR psychomotor skill competence. Resuscitation. 2009, 80:909-12.

10.1016/j.resuscitation.2009.04.021

7. Kirkpatrick DI: Evaluation of training. Training and Development Handbook. Craig R, Bittel I (ed): McGraw-Hill, New York, NY; 1967. 87-112.

8. American Board of Medical Specialities. MOC Competencies and Criteria . Accessed: 4/23/2014: http://www.abms.org/maintenance_of_certification/MOC_competencies.aspx. 